Journal of Engineering and Applied Sciences 15 (6): 1589-1597, 2020

ISSN: 1816-949X

(C) Medwell Journals, 2020

\title{
A New Deep Learning Method to Reconstruct and Estimate High Complex Features from the Presented MR Image
}

\author{
Nouf Saeed Alotaibi \\ Department of Computer Science, College of Science, Shaqra University, Shaqra, Saudi Arabia
}

\begin{abstract}
This study shows an effective deformable complex 3D image reconstruction and image synthesis technique by consolidating needed high-level features from Convolutional Neural Network (CNN) system. By recognize inherent deep feature representations in image patches for morphological changes in medicinal imaging information discovery. Utilizing the ADNI and LONI imaging datasets, image reconstruction and synthesis performance was verified with two existing design. Various performance measurements, High Frequency Error Norm (HFEN), Mean Squared Error (MSE), peak Signal-to-Noise Ratio (PSNR), Structural Similarity Index (SSI) are utilized to inspect different dataset. A deformable image reconstruction and synthesis strategy that uses conventional features has low value of MSE and HFEN. Likewise, to reveal the adaptability of the proposed image reconstruction and synthesis system, synthesis and reconstruction experiments were directed on 7T cerebrum MR image. As presented in the paper outcomes, the proposed method can accomplish predominant performance compared with other cutting-edge techniques with either low or high-level features in terms of the synthesis and reconstruction rate. In all investigations, the outcome shows that the proposed image synthesis and reconstruction framework reliably exhibited progressively precise outcomes when contrasted with best in class. Hence, it can be used for possible precise image reconstruction and synthesis related applications.
\end{abstract}

Key words: Fine-tuned deep learning, visualization, dynamic MR image reconstruction, MR image synthesis, framework

\section{INTRODUCTION}

Image reconstruction and deformable image synthesis has an enormous scope in flexible huge information applications, similar to medicinal image data processing and can be broadly divided into two type, idea based and contentbased strategies. Moreover, deformable image synthesis is essential to neuroscience and clinical examinations for normalizing individual subjects to the reference space (Schlemper et al., 2017). In deformable image registration, it is basic to build up exact anatomical correspondences between two medicinal images. With the advances of the Artificial Intelligence (AI) based processing strategy, Content-Based Image Retrieval (CBIR) has been additionally changed as multi-level with low-level and high-level features (Bahrami et al., 2016). The primary methodology includes manual ordering of images and along these lines it isn't attainable for an increased image dataset. Dot Diffuse Block Truncation Coding (DDBTC) which is a halftoning based Block Truncation Coding (BTC) force with good image quality, to obtain the essential features for productive image reconstruction (LeCun et al., 2015; Huynh et al., 2015; Zhang et al., 2015; Hofmann et al., 2008). To overcome the downside in DDBTC technique supervised processing strategies have been proposed. In supervised learning-based techniques to choose the best arrangement of features from a feature pool that may incorporate a lot of excess handmade features (Tisdall et al., 2012). In this case, the methodology, the ground-truth information with known correspondences over the arrangement of preparing pictures is required. Since, human specialists are normally expected to produce needed data, it is notable that getting this sort of information can be an extremely difficult and additional procedure needed. Conventionally, a patch based correspondence detection approach is frequently utilized where a patch is a fixed Region of Interest (ROI) symmetric neighbourhood of pixel intensity esteems focused a point in the image (Straub and Schulz, 2018; Huang et al., 2017; Yu et al., 2019). Also, if two patches, from two distinct images, show comparative same morphological ROI, the two values (at each patch focus) are viewed as related. Consequently, to improve correspondence identification, the issue turns into the one identified with feature choice, i.e. how to reliably choose a lot of highly discriminative features that can precisely and compactly, catch the morphological example displayed in the picture patch. Because deformable image synthesis is needed for presented input image, it commonly takes long duration to build up another image enlistment technique that has worthy execution for another imaging methodology or new imaging application. The conventional method for choosing features including the formation of similarity 
measurement but requires expert information that is logically identified with methodology and application. In this research work, we present a learning model to learn features from the presented complex medical image using unsupervised learning network. The principle contributions of this paper are:

We proposed a CNN Model to improve the medical image reconstruction and identify complex morphological region in $3 \mathrm{D}$ medical image patches through the combination of low-level and high-level features.

To improve up the matching, the CBIR and registration with synthesis structures sifting design is proposed. Feature dimension is diminished by PCA method for the inspected to deformable image synthesis on testing image modalities for classification.

Literature review: For the high-level features, Convolutional Neural Network (CNN) (also called as deep learning) is generally used for its viable feature descriptors and precise outcomes. The highlights created by profound learning mirror the human observation through different activities, for example, convolution and pooling, this make better component descriptors contrasted with the low-level model (Wang et al., 2018). In CNN, the previous architectures, AlexNet, GoogLeNet and VGG are some accurate architecture. Among these, AlexNet Model has improved inception module, requires less memory utilization and simple calculation, it is therefore, more famous than others (Doi, 2007; Arjovsky et al., 2017; Srivastava et al., 2014; Ghesu et al., 2017). However, in CNN module, it isn't ensured that the features from the most abnormal level can generally accomplish the best result. To avoid this issue, we proposed novel feature combination strategy which consolidates the low-level highlights from DDBTC and abnormal state AlexNet Completely Association Features (AN-CAF). Also, to diminish the feature dimension, the Principle Component Analysis (PCA) is utilized (Bengio et al., 2013). To avoid the identified above limitations, unsupervised learning-based feature selection method require alternate examination. Due to higher dimension nature of the information, traditional unsupervised methods that utilizes direct model, for example, ICA method are ordinarily not appropriate on the grounds that they are unfit to safeguard the data connections when used to the low-dimensional space. Also, since, the identified features are utilizing a shallow model, the identified features may need high-level discernment learning (e.g., shape and network data) and may use for ROI classification (James and Dasarathy, 2014; Guo et al., 2019; Shen et al., 2017; Wang et al., 2017). Now, unsupervised deep learning architecture determination systems have been effectively connected to solve advance computing problem. The concept for deep learning algorithms is first lower level features is learned and higher-level complex are presented to classify (Girshick et al., 2016). Moreover, the shallow models, deep learning engineering can encode complex data from easy to complex learning. Subsequently, for image enlistment, deep learning is promising in light of the fact that it it does not require prior knowledge, since, it is unsupervised model, utilizes a layered design to derive complex nonlinear connections is totally data driven and not depends on considered features and can rapidly and productively process the presented testing input data (Zhang et al., 2017).

\section{MATERIALS AND METHODS}

\section{Theporposed method used}

Problem formation: Autoencoder is efficient neural system and basically characterized by three consecutive layers: the processing layer, the hidden layer and output layer. Here, the objective of autoencoder is to understand image feature representations on the presented image patch. Give $\mathrm{W}$ and $\mathrm{H}$ is the dimension of presented discriminative image patch. Consider the presented input image patch $x_{i}=(n=1, \ldots, N)$, here, proposed DCNN is used to process the activation vector $F_{i}=\left[F_{i}(j)\right] i=1, \ldots, N$ with the activation function $\mathrm{M}(\mathrm{x})=\left(\mathrm{Wx}_{\mathrm{i}}+\mathrm{b}_{\mathrm{i}}\right)$, here weight vector has a dimension of $\mathrm{M} \times \mathrm{N}$. When we change the activation function to a bipolar sigmoidal function with discriminative value given by Eq. 1:

$$
\mathrm{S}(\mathrm{a})=\frac{1}{1+\mathrm{e}^{-\mathrm{a}}}
$$

Hence, the vector $F_{i}$ is considered as registration with synthesis vector of the presented input layer with proposed DCNN. Then, the discriminative image is presented to next hidden layer with a decoded norm vector $y_{m} \in \mathbb{R}^{L}$, this exactly register another deterministic value in the presented image patch. The weigh value and bias value calculated in this layer is given by mapping function $\mathrm{y}_{\mathrm{i}}=\left(\mathrm{W}^{\mathrm{T}} \mathrm{h}_{\mathrm{m}}+\mathrm{b}_{\mathrm{o}}\right)$. The energy function is given by:

$$
\left\{\mathrm{W}, \mathrm{b}_{\mathrm{i}}, \mathrm{b}_{\mathrm{j}}\right\}=\operatorname{argmax} \mathrm{M}(\mathrm{x})+\gamma\|\mathrm{y}-\mathrm{Sx}\|_{2}^{2}
$$

Figure 1 demonstrates the MRI dataset used for process in same uses. In Fig. 1, it is identified that same subject MRI has different appearances in any case, Evo-thing is implemented to separate both GL-FCF and Two-Layer Codebook include (TLCF) with various patches.

To identify the human level image understanding, high level feature is characterized with the presented image dataset. To reformulate the cost function from Eq. 2, we obtained the modified cost function as: 

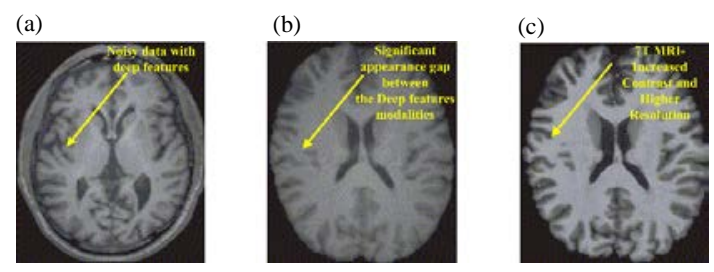

Fig. 1(a-c): Target images from same subject, (a) MRI brain image, (b) 3T brain MRI and (c) 7T brain MRI

$$
\operatorname{argmax} \mathrm{M}(\mathrm{x})+\rho\left\|\mathrm{y}-\mathrm{S}_{\mathrm{i}} \mathrm{x}\right\|+\varepsilon \| \mathrm{x}-\mathrm{y}||
$$

where, the value of penalty factor and cost function is varied according to mean distribution. The proposed cost function is computed using the linear iterative learning of cost function. The solution for AlexNet learning is given by:

$$
\begin{gathered}
\mathrm{X}_{\mathrm{i}+1}=\arg \min _{\mathrm{x}} \mathrm{M}(\mathrm{x})+\varepsilon \mathrm{x}^{\mathrm{i}+1} \\
\mathrm{Y}_{\mathrm{i}+1}=\arg \min _{\mathrm{y}} \mathrm{M}(\mathrm{x})+\varepsilon \mathrm{y}^{\mathrm{i}+1}
\end{gathered}
$$

To avoid the data consistency problem in proposed deep learning algorithm the closed form solution is considered for the modified data relativity:

$$
\begin{gathered}
X_{i}=\operatorname{DC}\left(x^{i} ; y, \rho\right)=S^{i+1}+\frac{\sigma}{1+\mu^{i}} \\
M_{x}= \begin{cases}1, & i<\gamma \\
\frac{1}{1+\mu}, & i \geq \gamma\end{cases}
\end{gathered}
$$

The proposed DCNN involve two important steps in designing. The procedure of feature preparing and synthesis from AlexNet Fully-Connection (AFC) is completely for image feature construction explained in section 3.2. In further, AFC feature extraction from DDBTC by the two layer codebook ordering is explained with its feature fusion for MRI synthesis from 3T to 7T MRI in section 3.3.

The feature from the fully associated AlexNet with $512 \times 512$ dimension is used in this investigation. The algorithm of high-level discriminative image feature extraction from proposed DCNN is presented as here: processing image data are resized to $512 \times 512$. To avoid overfitting problem and data residence, data of size $128 \times 128$ are identified from the entire image in random resized region. Then, the labelled dataset is presented into various convolution layers. The convolutional layer maps are changed by the Rectified Linear Units (ReLU). This ReLU act as bipolar sigmoidal activation function for the proposed DCNN. Hence, this method improves our overall efficiency. Local processing is carried for the resized $128 \times 128$ image patch. The parameter reduction is carried by max-pooling. Repeat the above steps until a fully connected output match. Deep feature representation: image synthesis and reconstruction calculation is an average element based deformable synthesis and reconstruction for MR images. We can use the high-quality characteristic vectors (i.e., the low level feature in multi dimension image patch) in the element based image synthesis technique. By the learned high-level feature component portrayals by the convolutional SAE to calculate the modified features between presented testing images.

Proposed feature construction technique: In previous technique, two primary features, bitmap and shading quantizers were acquired from DDBTC. In this manner, two different of dataset are formed. The component of VQ-recorded features is liable to the codebook length. While performing image reconstruction, the higher component of the dataset, the examination task of testing image with the image in the dataset takes longer time.

In this, in view of conventional architecture, we proposed the two-layer codebook processing architecture to classify the first formed codebook dataset. This makes a processing feature component with lower processing dimension which decrease the coordinating time among the retrieval of image. Moreover, it is a multi-dimensional codebook processing architecture with reduced processing time. The development of proposed classification algorithm is as per the following steps:

The presented feature and color quantizers are employed to generate original image codebooks dataset as described above, this shown in Fig. 2.

The size of the multi-layer codebook image processing architecture is determined and the image feature in the original dataset are processed. The random approach is to avoid separating similar color in the same direction of data classification.

Similar images are not guaranteed of the same class member, we randomly choose codewords to make sure that the all the codewords spread uniformly.

Finally, the two-layer codebook features are obtained by indexing two color quantizers and one presented image to two-layer classifier. Subsequently, we add up the counts from the different image to obtain needed feature. The above four steps are diagrammatically presented in Fig. 2.

Image synthesis architecture using deep learning feature representation: The trained DCNN with a large number of presented discriminative image patch, it is effective to get the reduced dimensional features using matrix product and summing with in each encoder layer. 


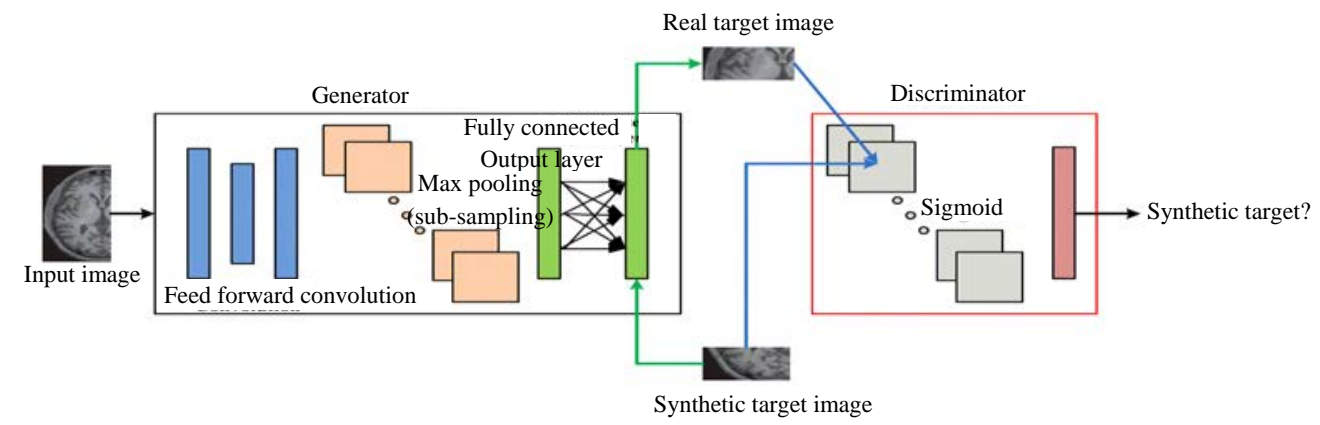

Fig. 2: Proposed deep convolution model for image synthesis estimation and reconstruction of target image using AlexNet architecture

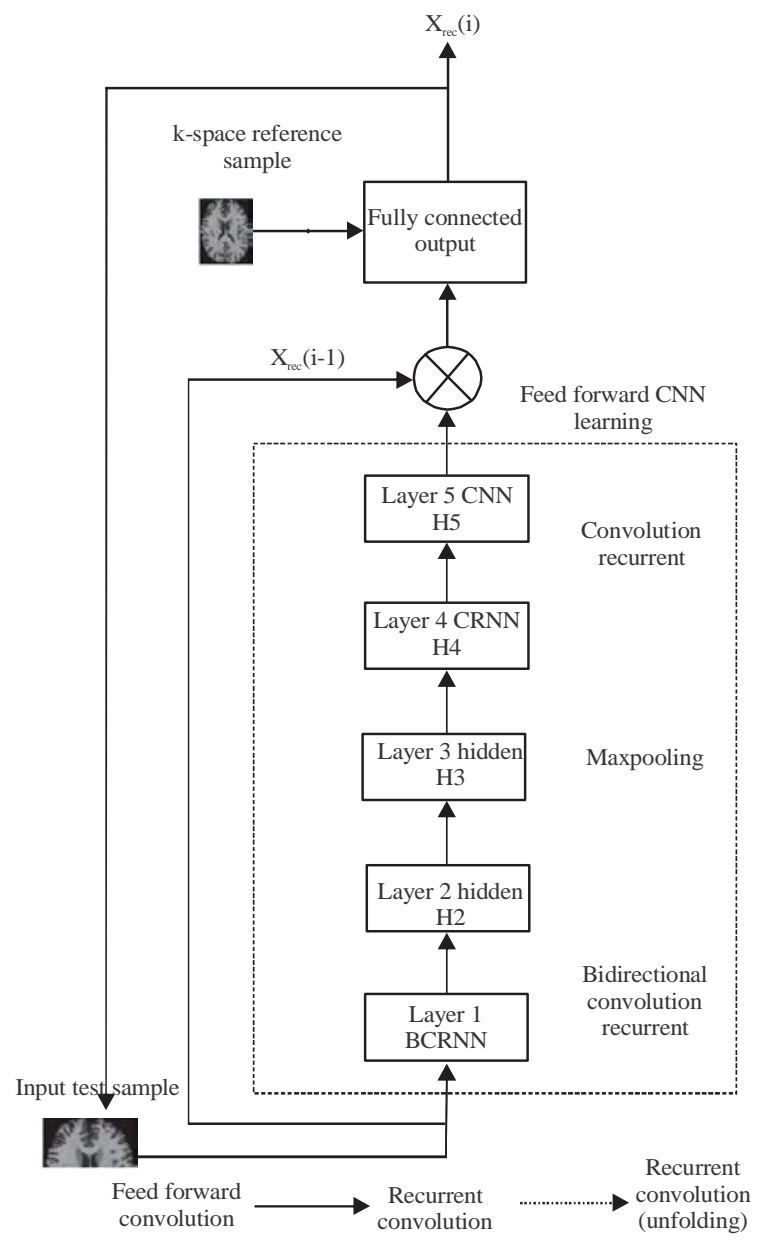

Fig. 3: Proposed AlexNet deep CNN overall architecture for MRI image reconstruction for unfolding iteration over the time sequence

The proposed deep learning architecture is developed based on TENSOR flow. From each image signature each point accurately identify the image registration. Also, in Fig. 3, the proposed deep convolution model for image synthesis estimation and reconstruction of target image using AlexNet architecture for image feature generator and discriminator is indicated with unfolding time samples.

Image sampling criteria using deep learning framework: Consider, a sample brain MR image with $1 \times 1 \times 1 \mathrm{~mm}^{3}$ resolution, the considered image patch has millions of small voxels in the entire assumed volume. Hence, not only the needed region is trained but also many numbers of unwanted image patches are trained with the proposed deep learning framework. To avoid this drawback an adaptive sampling with Boltzmann energy function is used for selecting image with higher discriminative image for the presented entire image. This procedure is used for image feature representation. For this objective the three design criteria for discriminative image patches are as follows:

For selected discriminative image patches for processing the region of interest should lies on sulcal of acquired MR images. Easy of identification distinctive regions are located in the entire dimension of the image. The considered dimension should cover entire MR image of brain.

Experiments and results: Our experimental verification dataset comprises of 10 subjects, each with 3T MRI $\left(1 \times 1 \times 1 \mathrm{~m}^{3}\right)$ and 7 T MRI $\left(0.65 \times 0.65 \times 0.65 \mathrm{~m}^{3}\right)$, examined utilizing 3T and 7T MRI scanners in two different conditions. The 7T MRI gives better resolution and differentiation than the 3T MRI in this way it provides benefits to early classification based onregion of interest. For processing these image data are arranged linear.

Network learning: Given the input data $\mathrm{N}$ of training target sets $\left(\mathrm{x}_{\mathrm{u}}, \mathrm{x}_{\mathrm{t}}\right)$, the classification model learning continues by limiting the pixel-wise Mean Squared Error (MSE) between the testing dataset and the reference dataset:

$$
\rho(\theta)=\frac{1}{\mathrm{n}} \sum_{\left(\mathrm{x}_{\mathrm{u}}, \mathrm{x}_{\mathrm{i}}\right)}^{\mathrm{n}}\left|\mathrm{x}_{\mathrm{i}}-\mathrm{-}_{\mathrm{x}}\right|^{2}
$$


where, $\theta=\left\{\mathrm{W}_{\mathrm{l}}, \mathrm{W}_{\mathrm{i}}, \mathrm{W}_{\mathrm{t}}, \mathrm{B}_{\mathrm{l}}\right\} \mathrm{l}=1, \ldots, \mathrm{L}$ and $\mathrm{n}_{\mathrm{s}}$ represents the total samples of testing dataset $S$. Here, total number of time sample $\mathrm{T}$ and iteration cycle steps $\mathrm{N}$ expected by the system before classification model specified in network architecture. The proposed CNN weights were initialized utilizing $\mathrm{H}_{\mathrm{e}}$ method and Adam optimizer presented it. In the proposed model training phase, sigmoidal activation function used in the region $[-5,5]$ to avoid the gradient explosion issue. The proposed system was executed in Python utilizing Theano and Lasagne libraries.

Dataset and implementation details: The proposed deep learning technique was assessed utilizing a complex 7T MR dataset comprising of 10 linearly arranged MR image. Each image consists of SSFP acquisition of 20 spatial and temporal images, these images contains $256 \times 256 \mathrm{~mm}$ field of view and $8 \mathrm{~mm}$ thickness. The processed dataset comprises of 24-channel data with testing framework measure $32 \times 32$ which manipulated by zero padding to the network estimate $128 \times 128$. Image sensitivity maps were standardized to a reference image and used to form a single multi-valued reconstructed image.

In evaluating experiments, the multi-valued images were back-propagated to recover kernel value. The presented dataset in input layer were obtained by under sampled image sequences were produced by arbitrarily under sampling the $\mathrm{k}$ space tests utilizing Cartesian under sampling value with under sampling dataset received from Eq. 5, for each image the eight least spatial frequencies were obtained and the data sampling distribution of k-space lines in the phase encoding was obtained by a zero-mean Gaussian conveyance. Here, the under sampling rates are expressed regarding the matrix size of image which is $128 \times 128$.

The experimental evaluation of proposed deep learning architecture for 3T MRI image reconstruction image with difference map and between is shown in Fig. 4, the difference map contains every dataset is processed by a seven layered network, consist of input layer with five hidden layers of convolution and max pooling. Finally, fully connected output layer presents the classification of the dataset. For all convolution and max pooling units, we consider a kernel size $\mathrm{k}=4$. The proposed CNN after the convolution layer proposed with multi valued complex image. For proposed the hidden layer with convolution stride of $\mathrm{m}=1$ and pooling of $n=3$. Finally, the fully connected sub-sampling region for output class mapping. In the training of network for an epoch of $\mathrm{N}=12$ and total sample sequence time of $\mathrm{T}=45 \mathrm{sec}$ data.

The performance assessment was done through a three different case cross verification where for two cases we train on 12 samples at that point test on 3 different

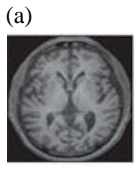

3T MRI

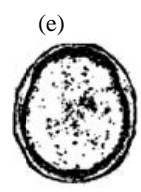

(b)

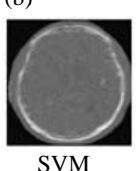

(c)

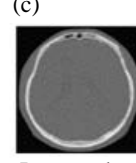

Proposed DCNN
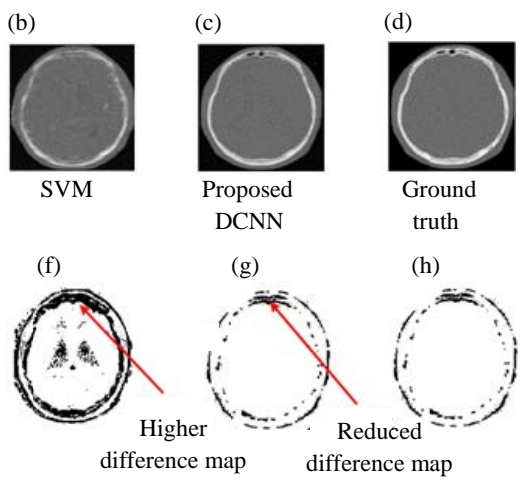

Fig. 4(a-h): Visualization results comparison of 3T MRI synthesis. First row presents comparison of MRI, SVM, DCNN and ground truth. Second row shows the target image difference map and between each corresponding estimated target comparison

subjects and for the last case of the data, we train on four subjects and test on 2 subjects. Here, the first dataset has dimension of $128 \times 128 \times \mathrm{T}$, for the learning phase, we identified a informative image patches of size $128 \times$ Dpatch $\times \mathrm{T}$ where Dpatch $=16$ is the dimension feature and the value of informative patch.

Discriminative image patch extraction and data dimension reduction affects the random error with elastic data transformation of the processed image. In Fig. 5, impact of using proposed method for image synthesis is shown. In Fig. 5, first row shows synthesis of 7T MRI using SVM, DCNN and proposed method, second row shows error difference map between synthesis of 7T MRI. In Fig. 5 second row it is clearly seen the value of proposed learning method produce clear reconstruction with equivalent to ground truth MR image.

Under sampling data masking were also performed additionally based on region of interest on the presented input data. In the testing phase, the trained deep CNN is applied for both discriminative and informative patches of the linearly arranged data sequence. The initial counter for processing algorithm begins with the $n=1$ and we considered the network performance by back propagations of error with hidden layers.

Evaluation method: Performance of proposed deep learning algorithm is compared and contrasted with the representative methods like SVM based dynamic MRI classifier, for example, k-t FOCUSS and one variations of CNN algorithm named network averaged 3D-CNN. The proposed deep CNN network shares a similar learning algorithm with the proposed AlexNet model, yet, all the alternating units and conventional CNN units were replaced with 3D convolutional units. The proposed architecture was compared with network parameter. We obtained, for proposed deep learning with AlexNet 
J. Eng. Applied Sci., 15 (6): 1589-1597, 2020

(a)

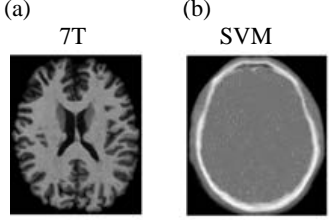

(e) Higher error difference map

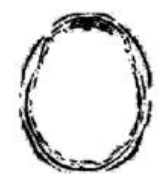

(c)

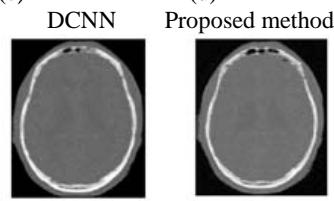

(f) Moderate error Lower error difference than difference ground truth (ground truth)

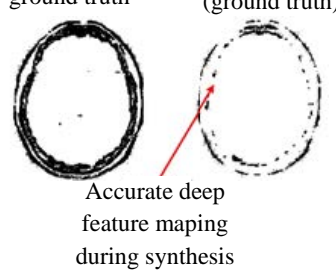

Fig. 5(a-g): Impact of using proposed method for image synthesis. First row shows synthesis of $7 \mathrm{~T}$ MRI using SVM, DCNN and proposed method. Second row shows error difference map between synthesis of 7T MRI

architecture to the system without weight sharing, wherein the system limit is $\mathrm{N}=5$ times of that of $3 \mathrm{D}$ CNN-S and roughly 6 times more than that of SVM based technique.

For the vector-based classifiers, the learning algorithm receptive field size is $7 \times 7 \times 7$ as this field used for memory reset in each successive layer. Compared with the proposed technique because of the fully connected output layer in the AlexNet learning model and bidirectional error transformation by using subsampling of the convolution layers engaged with forward error transmission including both spatial and temporal changes on direction in principle, the open field size is $210 \times 210 \times 17$. Image reconstruction results were assessed dependent on the accompanying quantitative measurements like High Frequency Error Norm (HFEN), Mean Squared Error (MSE), Peak Signal-to-Noise Ratio (PSNR), Structural Similarity Index (SSI). The decision of these measurements was made to assess the presented image reconstruction results with conventional classifiers. To verify the accuracy of image reconstruction MSE and PSNR values are used. The overall quality of image reconstruction is expressed by SSI. Error norm values are expressed for verification of quality in image reconstruction. In the proposed AlexNet Model with the layer filter kernel size $12 \times 12$ pixels. Higher the value of SSI and PSNR gives the better clarity also from the lower value of HFEN and MSE gives accurate image reconstruction.

\section{RESULTS AND DISCUSSION}

The performance evaluation on real time MRI reconstruction of proposed method with conventional

Table 1: Performance comparison on real time MRI reconstruction

\begin{tabular}{llll}
\hline & Classifier model used & \\
& - & - & \\
Parameters & SVM & 3D CNN & Proposed method \\
\hline SNR & 32.46 & 34.17 & 44.25 \\
MSE & 0.32 & 0.41 & 0.192 \\
HFEN & 0.56 & 0.172 & 0.15 \\
SSI & 0.91 & 0.85 & 0.97 \\
Training time & $65 \mathrm{sec}$ & $19 \mathrm{sec}$ & $5 \mathrm{sec}$ \\
\hline
\end{tabular}

classification method is presented in Table 1 where we assessed the image reconstruction time and quantitative measurements like MSE, SSI and HFEI. The average value of performance metrics with network training time is shown in Table 1. From the training time we can understand that the proposed method has lesser value for reconstruct the complex feature from the real time MR image.

The obtained values of performance index are shown in highlighted bold text. On comparing, the obtained value with the SVM based classifier, the proposed deep learning methods based on AlexNet outperform in terms of image reconstruction and synthesizes. The rate of capacity of image reconstruction based on acceleration rate of proposed method is increased by 33\% of that other image reconstruction method. From the value of SNR and HFEN the competitiveness and efficiency of proposed image, reconstruction method is verified. Furthermore, we can see a generous improvement of the image synthesis and reconstruction results on all dataset presented.

The total number of iterations utilized by the proposed network in the training phase is set in test phase also. For this AlexNet Model an iteration $\mathrm{N}=8$ is used for all the presented dataset. In addition, we have identified when training epoch number is changed up to $\mathrm{N}=10$, it gives an improvement of $0.425 \mathrm{~dB}$ on average SNR value. Figure 6 gives the diagrammatic presentation of performance with various values of testing on 7T MRI dataset iteration epoch. Correspondingly, image reconstruction in the intermediate iteration is given in Fig. 6 with SNR value comparison with other conventional method like SVM and 3D CNN method. In Fig. 6 where we can predict the steady improvement of the image synthesis and reconstruction value from each epoch count $N=1-10$ which is reliable with the quantitative outcomes of our proposed deep learning method.

Visualization result comparison of image reconstruction of the testing data in terms of MSE loss shown in Fig. 7 with the synthesized image, reconstruction of image and their error value in terms of MSE of various method compared. From this, we can identifies, our proposed deep learning model can deliver accurate reconstruction for those deep features of the images around the myocardium.

Regarding speed of image reconstruction, the proposed AlexNet architecture based deep learning model 


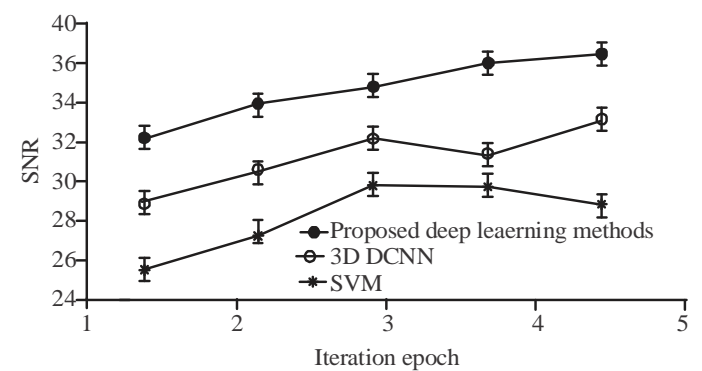

Fig. 6: Graphical comparison of SNR of proposed method and other methods on 7T MRI dataset with iteration epoch

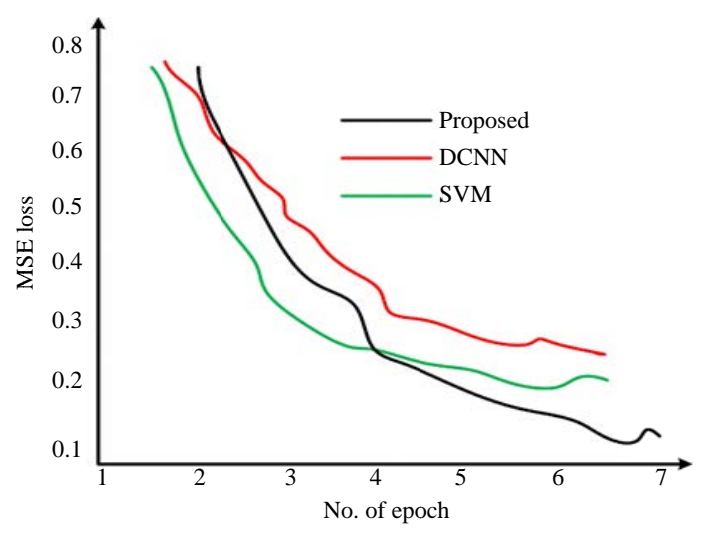

Fig. 7: Comparison of MSE of proposed method with other method of the generator in testing MRI Dataset for various epoch

reconstruct faster than the 3D-CNN and SVM based classifiers. Image reconstruction time of proposed deep learning model and the other vector-based techniques detailed in Table 2. By the proposed method mean standard deviation for 7T MR image synthesis from 3T is of 1.21 with a training time of 5 sec in 10 epochs. These results were obtained on a GPU DNVIDA GTX 1080, i7 processor with $1 \mathrm{~TB}$ memory space for real time processing of the testing dataset.

Discussion on image synthesis: In this experiment, we have shown that the proposed system is fit for creating accurate image synthesis and under sampled MRI image reconstruction, from the value of performance metrics and error map inspection. On comparing with the conventional databased learning methods, we have proposed an gatherable learning using AlexNet architecture and propagate the training error using back propagation algorithm. In Table 1, we can identifies compared with 3D CNN Models; the proposed technique has a much lower MSE error with higher SNR and SSI. This proves the increased accuracy and effectiveness of our deep learning algorithm. This is because of the capacity of the
Table 2: Result summarizing of our proposed deep learning model on 3T-7T MRI dataset with its mean standard deviation

\begin{tabular}{ll}
\hline Leaning method & Mean (Std.) \\
\hline SVM & $24.1(1.71)$ \\
3D CNN & $27.5(1.510$ \\
Proposed model & $29.4(1.21)$ \\
\hline
\end{tabular}

Table 3: Performance comparison of image synthesis on MRI dataset in terms of for various inputs

\begin{tabular}{llrrr}
\hline & & \multicolumn{3}{l}{ Classifier model used } \\
\cline { 2 - 5 } Input image & Parameters & ----------- \\
\hline MRI & SSI & \multicolumn{1}{c}{29.6} & \multicolumn{1}{c}{27.5} & 94.92 \\
dataset & MSE & 0.870 & 0.756 & 0.910 \\
Ground truth 3T MRI & SNR & 31.400 & 30.500 & 0.320 \\
& SSI & 0.710 & 0.820 & 0.960 \\
Synthesis 7T MRI & MSE & 0.910 & 0.650 & 0.270 \\
& SNR & 32.500 & 37.400 & 42.710 \\
& SSI & 0.810 & 0.940 & 0.972 \\
& MSE & 0.710 & 0.410 & 0.190 \\
\hline
\end{tabular}

proposed AlexNet units to build the open field measure while emphasis steps increment, just as to productively spread image data over the transient real time acquiring. For complex, image with higher undersampling rate at the real time image processing altogether, add noise to the initial image synthesis and reconstruction, this makes the image reconstruction of complex MRI as more challenging.

Performance comparison of image synthesis on MRI dataset in terms of for various objectives is shown in Table 3. For each objective different input are used as described in section 1. We have obtained an MSE of 0.27 and 0.19 for synthesis of ground truth to 3T MRI and 3T to 7T MRI, respectively. In addition, the proposed deep learning method has a high value of SNR for all the presented MR image dataset. This factor is considered for modelling the network to be suitable for complex dynamic medical image reconstruction with linear arrangement of data (Table 3). We can identify from the value of HFEN the vector-based method has higher denoise in the image background region. The proposed gatherable-based architecture produce improvement in the denoising of the background region of complex MRI data because of its larger receptive field sizes in the presented layer.

Moreover, when investigating the hidden layer feature reconstruction, we saw that the pair-wise classification and separations for proposed model were lesser for other constructing models like SVM and 3D-CNNs. In Fig. 8, we show the visual comparison with 3T MRI, synthetic 7T MRI cortical region. Second row indicates the error difference map between proposed method and other methods. In second row arrow indicate the accurate synthesis of 7T MR image particularly on sub cortical region which is a high complex to synthesis and reconstruct. In Table 3, comparison of performance index in terms of SSI for various model is presented. We hypothesize this is on the grounds data that proposed 


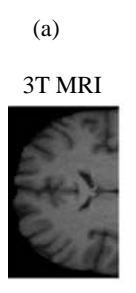

(e) Ground truth

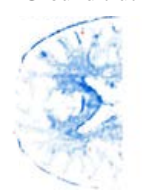

(b)

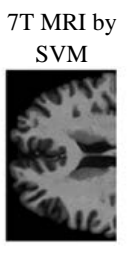

(f)

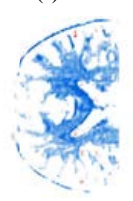

(c)

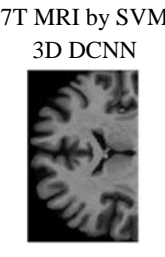

(g)

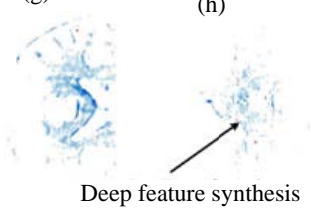

Fig. 8(a-h): Performance visual comparison with 3T MRI, synthetic 7T MRI cortical region. Second row indicates the error difference map between proposed method and other methods

AlexNet architecture based deep learning model has concealed associations over the dynamic MRI data using back propagation of error.

Moreover, SVM needs to additional computation for the learning of complex data in 3T MRI. This prove the proposed model is more sensitive for complex MRI reconstruction. Also, in the proposed model one can observe higher value of SSI 7T MRI synthesis due to smaller receptive region. This produce the higher accurate deep feature prediction in information.

\section{CONCLUSION}

In this research work, we have proposed a deep learning model from AlexNet architecture to reconstruct and estimate high complex features from the presented medical images. For evaluation of proposed model, a special performance index namely MSE, SSI and HSF are calculated. We have validated the proposed deep learning model by conducting experiment with three different task of MRI brain image reconstruction and synthesis namely; reconstruction and synthesis of 7T MRI from 3T MRI, predicting ground truth error in image reconstruction synthesis of 3T to 7T MRI image. The experiment conclude that highly complex feature image can reconstructed and synthesis with our proposed deep learning model. Moreover, the result demonstrates that it satisfactorily outperforms the other two advanced methods in same task with same datasets.

\section{REFERENCES}

Arjovsky, M., S. Chintala and L. Bottou, 2017. Wasserstein generative adversarial networks. Proceedings of the 34th International Conference on Machine Learning, July 7-10, 2017, London, UK., pp: 214-223.
Bahrami, K., F. Shi, X. Zong, H.W. Shin, H. An and D. Shen, 2016. Reconstruction of 7T-like images from 3T MRI. IEEE. Trans. Med. Imaging, 35: 2085-2097.

Bengio, Y., A. Courville and P. Vincent, 2013. Representation learning: A review and new perspectives. IEEE. Trans. Pattern Anal. Mach. Intell., 35: 1798-1828.

Doi, K., 2007. Computer-aided diagnosis in medical imaging: Historical review, current status and future potential. Comput. Med. Imaging Graphics, 31: 198-211.

Ghesu, F.C., B. Georgescu, Y. Zheng, S. Grbic, A. Maier, J. Hornegger and D. Comaniciu, 2017. Multi-scale deep reinforcement learning for real-time 3D-landmark detection in CT scans. IEEE. Trans. Pattern Anal. Mach. Intell., 41: 176-189.

Girshick, R., J. Donahue, T. Darrell and J. Malik, 2016. Region-based convolutional networks for accurate object detection and segmentation. IEEE. Trans. Pattern Anal. Mach. Intell., 38: 142-158.

Guo, Z., X. Li, H. Huang, N. Guo and Q. Li, 2019. Deep learning-based image segmentation on multimodal medical imaging. IEEE. Trans. Radiat. Plasma Med. Sci., 3: 162-169.

Hofmann, M., F. Steinke, V. Scheel, G. Charpiat and J. Farquhar et al., 2008. MRI-based attenuation correction for PET/MRI: A novel approach combining pattern recognition and atlas registration. J. Nucl. Med., 49: 1875-1883.

Huang, Y., L. Shao and A.F. Frangi, 2017. Cross-modality image synthesis via weakly coupled and geometry co-regularized joint dictionary learning. IEEE. Trans. Med. Imaging, 37: 815-827.

Huynh, T., Y. Gao, J. Kang, L. Wang, P. Zhang, J. Lian and D. Shen, 2015. Estimating CT image from MRI data using structured random forest and auto-context model. IEEE. Trans. Med. Imaging, 35: 174-183.

James, A.P. and B.V. Dasarathy, 2014. Medical image fusion: A survey of the state of the art. Inf. Fusion, 19: 4-19.

LeCun, Y., Y. Bengio and G. Hinton, 2015. Deep learning. Nature, 521: 436-444.

Schlemper, J., J. Caballero, J.V. Hajnal, A.N. Price and D. Rueckert, 2017. A deep cascade of convolutional neural networks for dynamic MR image reconstruction. IEEE. Trans. Med. Imaging, 37: 491-503.

Shen, D., G. Wu and H.I. Suk, 2017. Deep learning in medical image analysis. Annu. Rev. Biomed. Eng., 19: 221-248.

Srivastava, N., G. Hinton, A. Krizhevsky, I. Sutskever and R. Salakhutdinov, 2014. Dropout: A simple way to prevent neural networks from overfitting. J. Mach. Learn. Res., 15: 1929-1958. 
Straub, M. and V. Schulz, 2018. Joint reconstruction of tracer distribution and background in magnetic particle imaging. IEEE. Trans. Med. Imaging, 37: 1192-1203.

Tisdall, M.D., A.T. Hess, M. Reuter, E.M. Meintjes, B. Fischl and A.J.V.D. Kouwe, 2012. Volumetric navigators for prospective motion correction and selective reacquisition in neuroanatomical MRI. Magn. Reson. Med., 68: 389-399.

Wang, S., M. Zhou, Z. Liu, Z. Liu and D. Gu et al., 2017. Central focused convolutional neural networks: Developing a data-driven model for lung nodule segmentation. Med. Image Anal., 40: 172-183.

Wang, Y., L. Zhou, B. Yu, L. Wang and C. Zu et al., 2018. 3D auto-context-based locality adaptive multi-modality GANs for PET synthesis. IEEE. Trans. Med. Imaging, 38: 1328-1339.
Yu, B., L. Zhou, L. Wang, Y. Shi, J. Fripp and P. Bourgeat, 2019. Ea-GANs: Edge-aware generative adversarial networks for cross-modality MR image synthesis. IEEE. Trans. Med. Imaging, 38: 1750-1762.

Zhang, J., Y. Xia, Y. Xie, M. Fulham and D.D. Feng, 2017. Classification of medical images in the biomedical literature by jointly using deep and handcrafted visual features. IEEE. J. Biomed. Health Inf., 22: 1521-1530.

Zhang, Y., G. Zhou, J. Jin, X. Wang and A. Cichocki, 2015. Optimizing spatial patterns with sparse filter bands for motor-imagery based brain-computer interface. J. Neurosci. Methods, 255: 85-91. 